TRANSACTIONS OF THE

AMERICAN MATHEMATICAL SOCIETY

Volume 351, Number 7, Pages 2961-2978

S 0002-9947(99)02010-3

Article electronically published on March 1, 1999

\title{
EQUATIONS IN THE Q-COMPLETION OF A TORSION-FREE HYPERBOLIC GROUP
}

\author{
O. KHARLAMPOVICH, E. LIOUTIKOVA, AND A. MYASNIKOV
}

\begin{abstract}
In this paper we prove the algorithmic solvability of finite systems
\end{abstract} of equations over the $\mathbf{Q}$-completion of a torsion-free hyperbolic group.

It was recently proved in [1] that finite systems of equations over the $\mathbf{Q}-$ completion of a finitely generated free group are algorithmically solvable. In this paper we generalize the results of [1] to the case of the $\mathbf{Q}-$ completion $G^{\mathbf{Q}}$ of an arbitrary torsion-free hyperbolic group $G$ with generators $d_{1}, \ldots, d_{N}$. (A detailed definition of the $\mathbf{Q}$-completion of a group can be found in [1].)

Main Theorem. Let $G$ be a torsion-free hyperbolic group. Then there exists an algorithm that decides if a given finite system of equations over the $\mathbf{Q}$-completion of $G$ has a solution, and if it does, finds a solution.

By a triangular equation we mean an equation with at most three terms. Given a system $S$ of equations in $G^{\mathbf{Q}}$, we may assume that all equations in $S$ are triangular. Indeed, an arbitrary equation $x_{1}^{\varepsilon_{1}} x_{2}^{\varepsilon_{2}} \ldots x_{n}^{\varepsilon_{n}}=1$ (where $x_{i}$ stands either for a variable or for a constant, $\varepsilon_{i}= \pm 1$ ) is equivalent to a finite system of triangular equations: $x_{1}^{\varepsilon_{1}} x_{2}^{\varepsilon_{2}} y_{1}^{-1}=1, y_{1} x_{3}^{\varepsilon_{3}} y_{2}^{-1}=1, \ldots, y_{n-2} x_{n-1}^{\varepsilon_{n-1}} x_{n}^{\varepsilon_{n}}=1$. Adding a finite number of new variables and equations, we may also assume that $S$ contains only equations with coefficients in $G$. To achieve this, we replace every constant of the form $d^{\frac{m}{n}}$ by a new variable $z$ satisfying the equation $z^{m}=d^{n}$.

From now on, we fix a finite system $S$ of triangular equations over $G^{\mathbf{Q}}$ with coefficients in $G$. We will reduce the system $S$ to a finite set of systems in a specific hyperbolic group $G *\left\langle t_{1}, \ldots, t_{\psi(m)}\right\rangle$, where the number $\psi(m)$ can be determined effectively given the number $m$ of equations in the original system $S$. The resulting systems are accompanied by the restriction that some of the variables belong to a certain subgroup $G *\left\langle t_{1}, \ldots, t_{i}\right\rangle$ of $G *\left\langle t_{1}, \ldots, t_{\psi(m)}\right\rangle$, i.e. do not contain certain t's. To each of the systems we can apply the (slightly modified) method of Rips and Sela [4] to see if they are decidable. If none of them is consistent, then the original system $S$ has no solution; if at least one has a solution, then it is possible to find a corresponding solution to the system $S$.

Suppose that the system has a solution in $G^{\mathbf{Q}}$; let $\left\{X_{1}, \ldots, X_{L}\right\}$ be a solution with the minimal possible number of roots. Since the solution belongs to $G^{\mathbf{Q}}$, it is contained in a certain group $K$, obtained from $G$ by adding finitely many roots. It is the union of a chain of subgroups $H_{i}$ defined as follows. Let $G=H_{0}$.

Received by the editors August 14, 1996.

1991 Mathematics Subject Classification. Primary 20E05, 20F10.

The first author was supported by grants from NSERC and FCAR.

The third author was supported by the NSF grant DMS-9103098. 
Step 1. Consider pairwise nonconjugated cyclically minimal primitive elements $u_{1}, \ldots, u_{k_{1}} \in G,\left|u_{1}\right| \leq \ldots \leq\left|u_{k_{1}}\right|$ (here $|u|$ denote the length of $u$ in $G$ ), and add roots $t_{1}, \ldots, t_{k_{1}}$, such that $u_{j}=t_{j}^{s_{j}}$. (Notice that $u_{i+1}$ does not become a proper power after we add roots $t_{1}, \ldots, t_{i}$.) The corresponding groups are denoted by $H_{1}, \ldots, H_{k_{1}}$, where $H_{j+1}=H_{j} *_{u_{j+1}=t_{j+1}}^{s_{j+1}}\left\langle t_{j+1}\right\rangle$.

Step 2. Consider pairwise nonconjugated primitive elements $u_{k_{1}+1}, \ldots, u_{k_{2}} \in H_{1}$, cyclically reduced in the amalgamated product, each having the reduced form $u=$ $t_{1}^{\alpha_{1}} c_{1} \ldots t_{1}^{\alpha_{k}} c_{k}$, where $\alpha_{i} \neq 0, \alpha_{i} \in \mathbf{Z}, c_{i} \in G,\left|u_{k_{1}+1}\right|_{H_{1}} \leq \ldots \leq\left|u_{k_{2}}\right|_{H_{1}} ;$ and add roots $t_{k_{1}+1}, \ldots, t_{k_{2}}$, such that $u_{j}=t_{j}^{s_{j}}$, to the group $H_{k_{1}}$. The corresponding groups are denoted by $H_{k_{1}+1}, \ldots, H_{k_{2}}$, where $H_{j+1}=H_{j} *_{u_{j+1}=t_{j+1}^{s_{j+1}}}\left\langle t_{j+1}\right\rangle$.

Step i+1. Suppose that $H_{1}, \ldots, H_{k_{i}}$ have been constructed.

Consider pairwise nonconjugated primitive elements $u_{k_{i}+1}, \ldots, u_{k_{i+1}} \in H_{i}$, cyclically reduced in the amalgamated product, each having the reduced form $t_{i}^{\alpha_{1}} c_{1} \ldots$ $t_{i}^{\alpha_{k}} c_{k}$, where $\alpha_{i} \neq 0, \alpha_{i} \in \mathbf{Z}, c_{i} \in H_{i-1}$ ( $c_{k}$ is not a power of $u_{i}$, because the elements are cyclically reduced), $\left|u_{k_{i}+1}\right|_{H_{i}} \leq \ldots \leq\left|u_{k_{i+1}}\right|_{H_{i}}$, and add roots $t_{k_{i}+1}, \ldots, t_{k_{i+1}}$, such that $u_{j}=t_{j}^{s_{j}}$, to the group $H_{k_{i}}$. The corresponding groups are denoted by $H_{k_{i}+1}, \ldots, H_{k_{i+1}}$, where $H_{j+1}=H_{j} *_{u_{j+1}=t_{j+1}^{s_{j+1}}}\left\langle t_{j+1}\right\rangle$.

Finally, for some number $i$ one has $K=H_{k_{i+1}}$.

The group $H_{k_{i+1}}$ is called the group at level $i$, corresponding to the sequence $u_{1}, \ldots, u_{k_{i+1}}$. The group $H_{i}$ will be called the group of rank $i$. We also order the set of $t_{j}$ 's: $t_{k}<t_{l}$ if $k<l$.

Let $F_{i}$ be the free group with the same generating set as $H_{i}, i=1, \ldots, k_{1}$, and let $\beta: H_{i} \rightarrow F_{i}$ be a section, i.e. a mapping of sets such that $\pi \circ \beta=$ id. For our purposes, $\beta$ has to be of specific form; we will define it explicitly in section 2 . For $X \in H_{i}$ we will call $\beta(X)$ the canonical representative of $X$.

Definition 1. By an equational diagram over a group $H_{i}$ we mean an equation $X_{1} \ldots X_{n}=1$ together with a solution $A_{1}, \ldots, A_{n}$ and a diagram over $H_{i}$ having $\beta\left(A_{1}\right) \ldots \beta\left(A_{n}\right)$ as its boundary label. An equational triangle is an equational diagram corresponding to a triangular equation. A system of equational diagrams is a system of equations together with the system of diagrams such that the solution associated to each equational diagram is a solution of the whole system.

A free equational diagram is an equational diagram with no cells; the corresponding equations are called free equations.

If the level $i$ of the group $H_{k_{i+1}}$ is greater than zero, we can use the method of [1] to reduce each equation of the system $S$ to a bounded number of free equations and at most one equation over the group in the previous rank. We can proceed in the same way until we reach level 0 (i.e. the group $H_{k_{1}}$ ), for which we need to develop a separate method. In what follows we will denote $H_{k_{1}}$ by $H$.

\section{Some properties of the Cayley graph of $H$}

We will frequently use the following lemma:

Lemma 1. ([3, Lemma 1.5]) For each geodesic triangle $\left[x_{1}, x_{2}, x_{3}\right]$ in a $\delta$-space, there are points $y_{i} \in\left[x_{i-1}, x_{i+1}\right]$ (indices are considered modulo 3) such that

$$
d\left(x_{i}, y_{i-1}\right)=d\left(x_{i}, y_{i+1}\right)=\left(x_{i-1} \cdot x_{i+1}\right)_{x_{i}},
$$




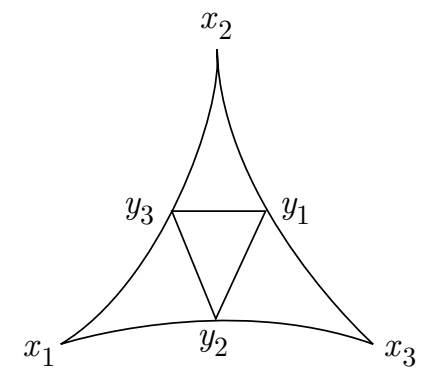

Figure 1

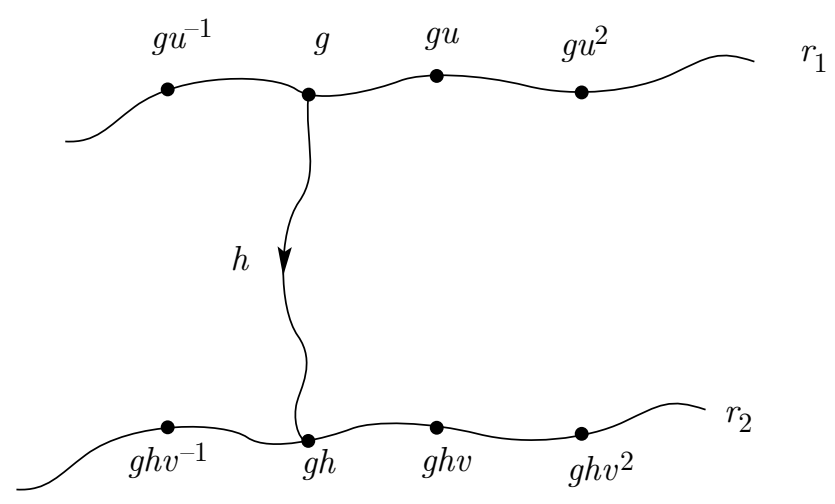

FIGURE 2

$$
d\left(y_{i}, y_{i-1}\right) \leq 4 \delta \text { and } d\left(u,\left[x_{i}, y_{i \pm 1}\right]\right) \leq 4 \delta
$$

for any point $u \in\left[x_{i}, y_{i \pm 1}\right]$. We will call the triangle $\left[y_{1}, y_{2}, y_{3}\right]$ the Gromov triangle inscribed in $\left[x_{1}, x_{2}, x_{3}\right]$ (see Fig. 1).

Lemma 2. [3, Lemma 1.9] There exists a constant $\mu=\mu(\delta, \lambda, c)$ such that for any $(\lambda, c)$-quasigeodesic path $p$ in a $\delta$-space and any geodesic path $q$ with the same initial and terminal points as $p$, the inequalities $d(Q, p)<\mu$ and $d(P, q)<\mu$ hold for any points $Q \in q$ and $P \in p$.

Definition 2. Let $u, v \in\left\{u_{1}, \ldots, u_{k_{1}}\right\},|u|_{G} \geq|v|_{G}$. In $\Gamma(H)$ consider two paths: a $u$-path $r_{1}$ and a $v$-path $r_{2}$, where $r_{1}$ connects the sequence of vertices $\ldots, g u^{-1}, g$, $g u, g u^{2}, \ldots, r_{2}$ connects the sequence of vertices $\ldots, g h v^{-1}, g h, g h v, g h v^{2}, \ldots$, $h \in H, g \in G$. (See Fig. 2.) Then a path $q$ that connects $r_{1}$ and $r_{2}$ is called a minimal path if

1. the image in $H$ of the label of $q$ is an element in reduced form in all ranks from 0 to $k_{1}$ (see [1] for terminology);

2. for each subpath $q^{\prime}$ of $q$ connecting $t$-syllables, the image of the label of $q^{\prime}$ is a geodesic word in $G$ and has minimal length among all words connecting the corresponding $t$-strips.

Definition 3. Let $d(P, q)$ denote the distance between a point $P$ and a path $q$ in the Cayley graph of $G$. Then a point $P^{\prime}$ is called the projection of $P$ onto $q$ if $P^{\prime} \in q$ and $d\left(P, P^{\prime}\right)=d(P, q)$. 


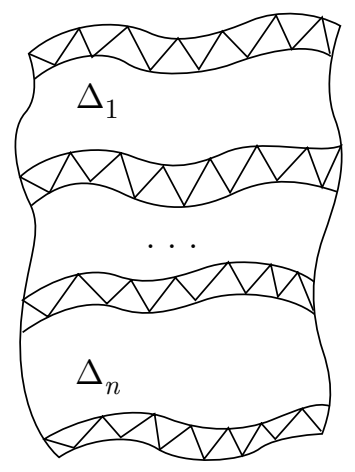

Figure 3

a)

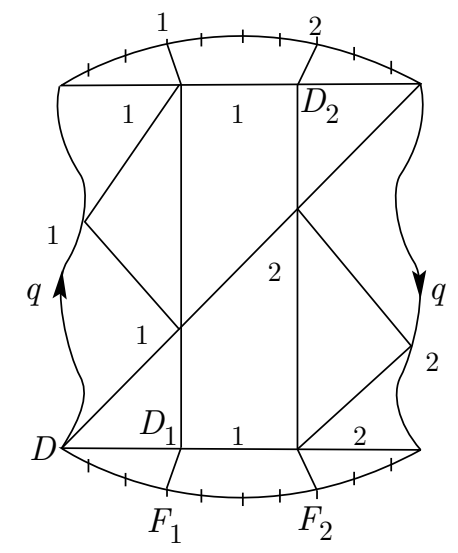

b)

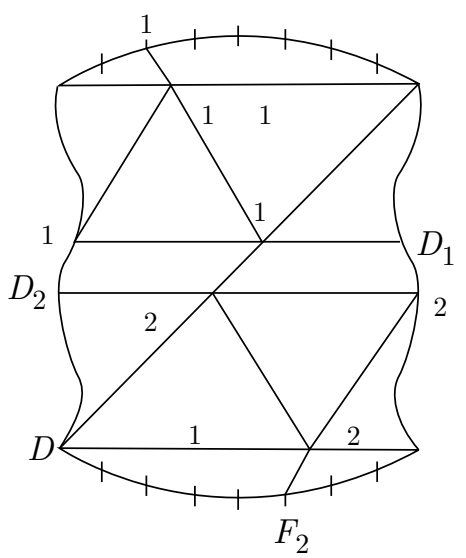

FiguRE 4

The following lemma says that the initial (resp. terminal) points of any two minimal paths are, in a sense, close to each other.

Lemma 3. Let $u, v, r_{1}, r_{2}$ be as in Definition 2, with $r_{1}$ and $r_{2}$ distinct; denote by $U$ the cyclic subgroup of $G$ generated by $u$, and by $V$ the cyclic subgroup generated by $v$. Let $q$ and $q^{\prime}$ be two minimal paths connecting $r_{1}$ and $r_{2}$, $X$ the u-periodic word corresponding to the subpath of $r_{1}$ connecting the initial points of $q$ and $q^{\prime}$, and $Y$ the $v$-periodic word corresponding to the subpath of $r_{2}$ connecting the terminal points of $q$ and $q^{\prime}$; i.e.,

$$
q^{-1} X q^{\prime}=Y
$$

Then there is a constant $M=M(G, U, V,|u|,|v|)$ such that

$$
|X|_{G},|Y|_{G} \leq M
$$

Proof. Whenever the context allows us to do so, we will often use the same notation for a path in $\Gamma(H)$ and the corresponding word in $H$.

We may assume that $q$ and $q^{\prime}$ do not contain any $t_{i}$ 's (otherwise subdivide the diagram corresponding to the equality $q^{-1} X q^{\prime}$ into several subdiagrams $\Delta_{1}, \ldots, \Delta_{n}$ 
with side labels containing no $t$-syllables as shown in Fig. 3; for each of them obtain a constant $M_{i}$; take $M$ to be $\left.\max \left(M_{1}, \ldots, M_{n}\right)\right)$.

Observe that the subgroups $U$ and $V$ are quasi-isometrically embedded in $G$; therefore, the paths corresponding to $X$ and $Y$ are $(\lambda, c)$-quasigeodesic for some $\lambda>0$ and $c \geq 0$ (see [3]). Let $X_{1}$ and $Y_{1}$ be the geodesic paths having the same initial and terminal points as the quasigeodesic paths $X$ and $Y$.

In the geodesic triangle $A B C D$, draw the diagonal $B D$. Inside each of the resulting triangles consider the Gromov triangles from Lemma 1. The triangles can be located with respect to each other in two different ways, as shown in Fig. 4 a) and b).

Case a. Let $D_{1}$ be the projection on $C_{1}$ onto $B_{2} D$ and $D_{2}$ the projection of $C_{2}$ onto $B_{1} B$. Then by Lemma $1 d\left(B_{1}, C_{1}\right) \leq 4 \delta, d\left(C_{1}, D_{1}\right)<4 \delta, d\left(B_{2}, C_{2}\right) \leq 4 \delta$, $d\left(C_{2}, D_{2}\right)<4 \delta$; therefore

$$
\left|B_{1} D_{1}\right|<8 \delta, \quad\left|B_{2} D_{2}\right|<8 \delta .
$$

Now take the projection of $B_{1}$ onto $X$ and let $E_{1}$ be the phase vertex on $X$ closest to this projection; in the same way, obtain the phase vertices $E_{2} \in X$ and $F_{1}, F_{2} \in Y$. Then $d\left(B_{1}, E_{1}\right), d\left(D_{1}, F_{1}\right), d\left(D_{2}, E_{2}\right), d\left(B_{2}, F_{2}\right)$ are all less than $\mu+\max (|u|,|v|)$. If $p$ is the path connecting $E_{1}$ with $F_{1}$ and $p^{\prime}$ the path connecting $E_{2}$ with $F_{2}$, then $|p|,\left|p^{\prime}\right| \leq 8 \delta+2(\mu+\max (|u|,|v|))$.

If $u \neq v$, the subgroups $U$ and $V$ satisfy the conditions of Lemma 4 from [2]: they are quasi-isometrically embedded in $G$, malnormal, and $U \cap g^{-1} V g=\emptyset$ for all $g \in G$ by the choice of $u$ and $v$. Therefore, there is a constant $M_{0}=M_{0}(G, U, V)$ such that either $\max \left(\left|E_{1} E_{2}\right|,\left|F_{1} F_{2}\right|\right)<M_{0}$ or $4 \max \left(|p|,\left|p^{\prime}\right|\right) \geq\left|E_{1} E_{2}\right|,\left|F_{1} F_{2}\right|$. Since in our case $p$ and $p^{\prime}$ have bounded length, it follows that $\left|E_{1} E_{2}\right|,\left|F_{1} F_{2}\right| \leq$ $\max \left(M_{0}, 4(8 \delta+2(\mu+\max (|u|,|v|)))\right.$.

If $u=v,\left|E_{1} E_{2}\right| \geq M_{0}$, then by the same lemma either $\left|E_{1} E_{2}\right|,\left|F_{1} F_{2}\right| \leq$ $4 \max \left(|p|,\left|p^{\prime}\right|\right)$ or $p, p^{\prime}$ represent powers of $u$. In the latter case, the paths $r_{1}$ and $r_{2}$ coincide, which is the case that we are not interested in.

It remains to bound $A E_{1}, B E_{2}, C F_{2}$ and $D F_{1}$.

Observe that $\left|E_{1} B_{1} A_{1}\right| \leq 4 \delta+\mu+|u|$; if $A A_{1}$ had greater length, then $A A_{1} D$ would not be a minimal path: $E_{1} B_{1} A_{1} D$, also connecting $r_{1}$ with $r_{2}$, would be shorter. Therefore, $\left|A A_{1}\right| \leq 4 \delta+\mu+|u|$, and $\left|A E_{1}\right| \leq\left|A A_{1}\right|+\left|E_{1} B_{1} A_{1}\right| \leq$ $8 \delta+2 \mu+2|u|$, as required. The same argument applies to the remaining paths.

Case b. Let $D_{1}$ be the projection of $C_{1}$ onto $B A_{2}, D_{2}$ the projection of $C_{2}$ onto $D A_{1}$. Observe that $\left|E_{1} B_{1} C_{1} D_{1}\right|=\left|E_{1} B_{1}\right|+\left|B_{1} C_{1}\right|+\left|C_{1} D_{1}\right| \leq \mu+|u|+8 \delta$. Therefore, $\left|B D_{1}\right| \leq \mu+|u|+8 \delta$ (otherwise $B C=q^{\prime}$ is not a minimal path), and $\left|E_{1} B\right| \leq\left|E_{1} B_{1} C_{1} D_{1}\right|+\left|B D_{1}\right| \leq 2(\mu+|u|+8 \delta)$. In a similar way, $\left|A E_{1}\right| \leq 2(\mu+|u|+4 \delta)$, which implies

$$
|X| \leq 4 \mu+4|u|+24 \delta .
$$

The same argument works for $Y$.

It follows from the above that the constant $M$ can be taken equal to

$$
\max \left(M_{0}, 4(8 \delta+2(\mu+\max |u|,|v|))\right)+24 \delta+4 \mu+4|u| .
$$

Therefore, for $u, v \in\left\{u_{1}, \ldots, u_{k_{1}}\right\}$, a $u$-path $r_{1}$ and a $v$-path $r_{2}$ from Definition 2 there are subpaths $r_{1}^{\prime} \subset r_{1}$ and $r_{2}^{\prime} \subset r_{2}$ such that $\left|r_{1}^{\prime}\right|,\left|r_{2}^{\prime}\right| \leq M$, the initial 


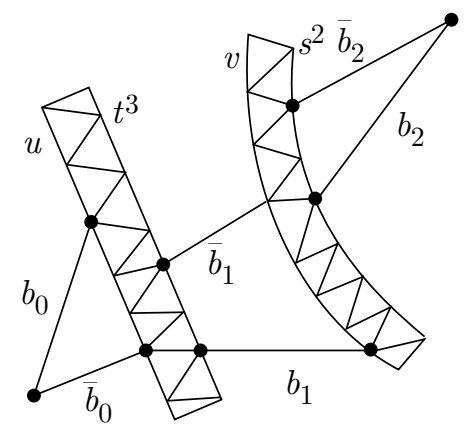

FiguRE 5

points of all minimal paths connecting $r_{1}$ and $r_{2}$ belong to $r_{1}^{\prime}$, and the terminal points, to $r_{2}^{\prime}$.

Definition 4. Following the terminology of [1], we call a minimal path between $r_{1}$ and $r_{2}$ a $(u, v)$-pseudoconnector for $h$. Let $q$ be a $(u, v)$-pseudoconnector, $p_{1}=\iota(q)$, $p_{2}=\tau(q)\left(p_{1} \in r_{1}^{\prime}, p_{2} \in r_{2}^{\prime}\right)$. Consider the two phase vertices $b_{1}$ and $b_{2}$ closest to $p_{2}$ on each side of $p_{2}$ on the $v$-path $r_{2}$. The connecting zone for $h$ with respect to $g$ is the union of all phase vertices between such $b_{1}$ and $b_{2}$ for all $(u, v)$-pseudoconnectors for $h$. Denote the initial vertex of the connecting zone by $(h)_{1}$ and the terminal vertex by $(h)_{2}$.

\section{Construction of Canonical Representatives}

Let $F$ be the free group with the same set of generators $d_{1}, \ldots, d_{N}$ as $G$; let $F_{i}=F *\left\langle t_{1}, \ldots, t_{i}\right\rangle$. For $0 \leq i \leq k_{1}$ we will define a section $\beta: H_{i} \rightarrow F_{i}$ (a mapping of sets such that $\pi \circ \beta=i d$ ). For $X \in H_{i}$ we will call $\beta(X)$ the canonical representative of $X$. (See [1] for the construction of canonical representatives for elements in the groups of ranks higher than $k_{1}$. )

The canonical representative of an element $X \in G$ is simply a fixed geodesic word representing $X$. If $X$ is an element of $H_{i}$ for $1 \leq i \leq k_{1}$ and $X$ contains $t_{i}$, then it can be written in the form $X=b_{0} t_{i_{1}}^{\alpha_{1}} b_{1} t_{i_{2}}^{\alpha_{2}} \cdots t_{i_{n}}^{\alpha_{n}} b_{n}$, where $i_{j} \in\{1, \ldots, i\}$, $b_{j} \in G$. By $\bar{b}_{j}$ we denote the canonical representative of the path connecting the vertices $\left(b_{j}^{-1}\right)_{2}$ and $\left(b_{j}\right)_{2}$; we will call $\bar{b}_{j}$ the $(u, v)$-pseudoconnector for the element $b_{j}$ (here $u=u_{j-1}, v=u_{j}$ ). The points $\left(b_{j-1}\right)_{2}$ and $\left(b_{j}^{-1}\right)_{2}$ can be connected by a path with label $t_{i_{j}}^{\gamma_{j}}$. Then the canonical representative of $X$ is defined to be $\bar{b}_{0} t_{i_{1}}^{\gamma_{1}} \bar{b}_{1} t_{i_{2}}^{\gamma_{2}} \ldots \bar{b}_{n}$

In the example illustrated in Fig.5, $X=b_{0} t^{11} b_{1} s^{-7} b_{2}$, where $u, v \in\left\{u_{1}, \ldots, u_{k_{1}}\right\}$, $u=t^{3}, v=s^{2}$. Then $\beta(X)=\beta\left(\overline{b_{0}}\right) t^{-5} \beta\left(\overline{b_{1}}\right) s^{-3} \beta\left(\overline{b_{2}}\right)$.

Below, all the elements $u_{j}$ 's are always represented by the words $\beta\left(u_{j}\right)$ and we will write $u_{j}$ instead of $\beta\left(u_{j}\right)$. It will be clear from the context when $u_{j}$ means a word and when it means the element represented by this word. 


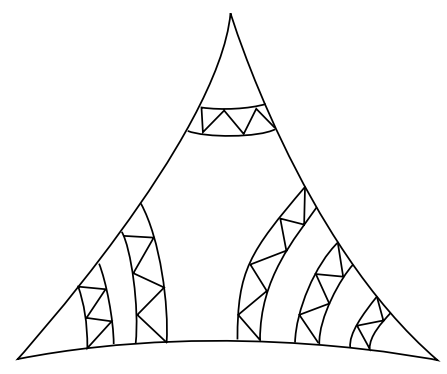

FigURE 6

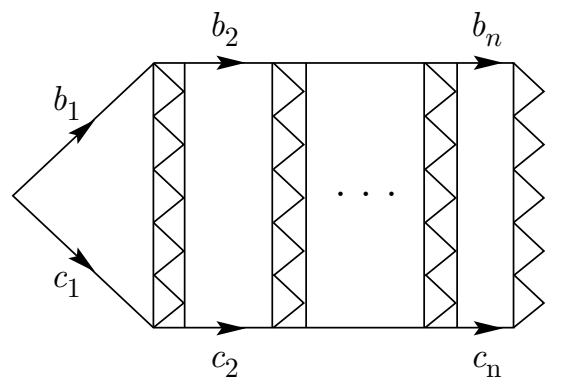

FIGURE 7

\section{Middles}

Let $X=\left\{X_{1}, \ldots, X_{L}\right\}$ be a solution of the system $S$ in the group $G^{\mathbf{Q}}$. Suppose that this solution is minimal, i.e. contains the minimal possible number of roots. Then for some $i$ the solution $X$ belongs to the group $H_{k_{i+1}}=K$.

For the solution $X$ we will construct another solution $X_{1}^{\prime}, \ldots, X_{L}^{\prime}$ and a system of equations over the group $G *\left\langle t_{1}, \ldots, t_{k_{i+1}}\right\rangle$ such that $\beta\left(X_{1}^{\prime}\right), \ldots, \beta\left(X_{L}^{\prime}\right)$ will be a part of a solution of this new system, and every solution of the new system will give a solution of the system $S$.

Here we will deal only with equational triangles in the group $H=H_{k_{1}}$, since for the higher ranks we can use the reduction method developed in [1].

Consider an equational triangle in $H$ with at least one side label containing $t_{i}$ for some $i \in\left\{1, \ldots, k_{1}\right\}$ (hence at least two side labels containing $t_{i}$ ). It is represented by a diagram of the form shown in Fig. 6. Note that the $t$-strips in Fig. 6 may correspond to distinct $t$ 's; a $t_{i}$-strip can start on a side of the equational triangle, but not on a $u_{j}$-side of a $t_{j}$-strip, since $u_{j} \in G$ and hence doesn't contain any $t$ 's.

The following lemma is a direct consequence of the construction of canonical representatives; it is an analog of Lemma 3 from [1].

Lemma 4. Suppose that in $H$ we have a diagram (see Fig. 7) with the boundary label $\left(b_{1} t_{j_{1}}^{r_{j_{1}}} \ldots t_{j_{n}}^{r_{j_{n}}} b_{n} t_{j_{n+1}}\right) t_{j_{n+1}}^{r_{j_{n+1}}}\left(c_{1} t_{j_{1}}^{p_{j_{1}}} \ldots t_{j_{n}}^{p_{j_{n}}} c_{n} t_{j_{n+1}}\right)^{-1}$, where $j_{1}, \ldots, j_{n+1}$ $\in\left\{1, \ldots, k_{1}\right\}, b_{1}, c_{1}, \ldots, b_{n}, c_{n} \in H$. Then

$$
\beta\left(b_{1} t_{j_{1}}^{r_{j_{1}}} \ldots t_{j_{n}}^{r_{j_{n}}} b_{n} t_{j_{n+1}}\right) t_{j_{n+1}}^{r_{j_{n+1}}}=\beta\left(c_{1} t_{j_{1}}^{p_{j_{1}}} \ldots t_{j_{n}}^{p_{j_{n}}} c_{n} t_{j_{n+1}}\right) .
$$

It follows from Lemma 4 that every equational triangle either does not contain any cells or takes on the form shown in Fig. 8 and hence has a unique maximal 


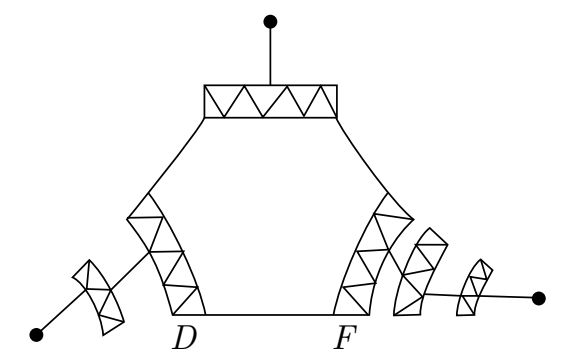

Figure 8

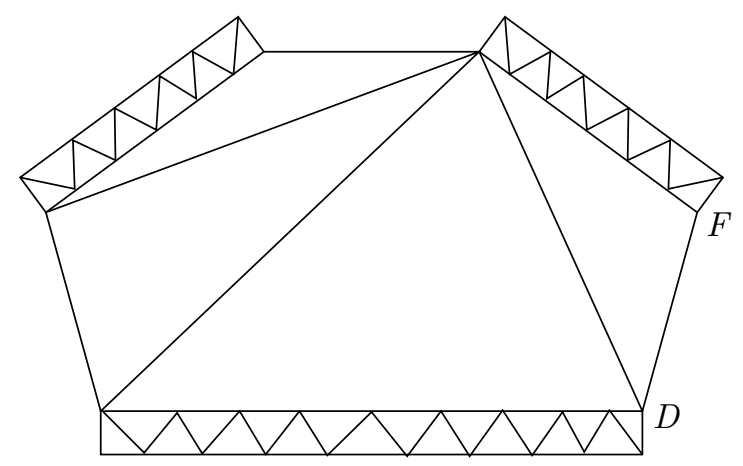

FiguRE 9

nontrivial $G$-subdiagram. (Some of the $t$-strips in Fig. 8 might, of course, be trivial.)

Definition 5. Consider an equational triangle $\Delta$ in $\Gamma\left(H_{i}\right)\left(1 \leq i \leq k_{1}\right)$ with the boundary label $\beta\left(X_{1}\right) \beta\left(X_{2}\right) \beta\left(X_{3}\right)$. The maximal nontrivial $G$-subdiagram of $\Delta$ is called the middle of this triangle. For example, the subdiagram $A B E F D C$ in Fig. 8 is a middle.

Let $u \in\left\{u_{1}, \ldots, u_{k_{1}}\right\}, t \in\left\{t_{1}, \ldots, t_{k_{1}}\right\}$. A boundary of a middle is canonically subdivided into paths. Each of these paths is either a $u$-path joining two phase vertices (i.e. belongs to the $u$-side of a $t$-strip) or a connector. The $u$-paths are called pseudoangles of the middle.

Let $\nu=\max \left\{\nu_{1}, \ldots, \nu_{8}\right\}$, where $\nu_{i}$ are certain fixed constants depending on the group $G$ and $u_{1}, \ldots, u_{k_{1}}$; we will see how to calculate the $\nu_{i}$ 's in the proof of Lemma 5.

A pseudoangle $p$ is said to be trivial if the corresponding $u$-path is trivial; short if $|p| \leq \nu$; long otherwise. A middle is called triangular if all the pseudoangles are trivial.

If none of $X_{i}$ 's contains $t$ then the middle of the triangle coincides with the triangle itself and is a triangular middle.

As an example, consider the middle $A B E F D C$ in Fig. 8. The paths $A B, C D$, and $E F$ are pseudoangles of this middle.

Each middle represents an equation over the group $G$; denote the corresponding system of equational diagrams by $\widetilde{S}$. 
a)

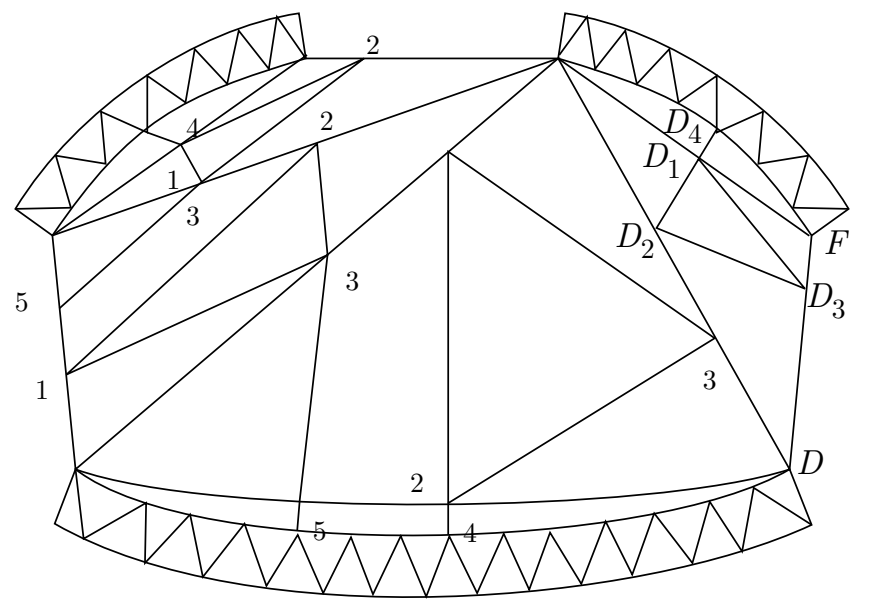

b)

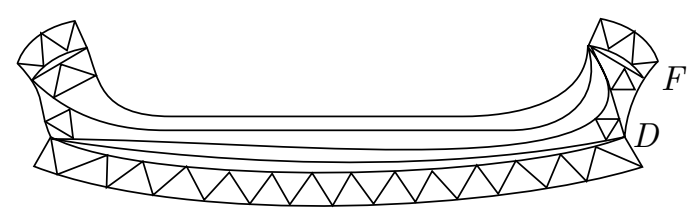

Figure 10

Lemma 5. Let $M$ be a middle of an arbitrary equational triangle over $H$. Then at most one pseudoangle of $M$ is long.

Proof. We will provide a proof for the case where none of the pseudoangles is trivial. Take an arbitrary triangulation of the geodesic hexagon obtained from the middle $M$ by replacing the pseudoangles, which are quasogeodesic paths, by the corresponding geodesic paths. We will use the triangulation shown in Fig. 9. Here $A B$ is a $u$-path, $C D$ is a $v$-path and $E F$ is a $w$-path for $u, v, w \in\left\{u_{1}, \ldots, u_{k_{1}}\right\}$. Inside each of the resulting triangles $A B E, A C E, C E D$ and $D E F$ consider the Gromov triangles from Lemma 1. These Gromov triangles can be arranged with respect to each other in eight different ways. Instead of providing a detailed proof for each of the eight possibilities, we will consider two typical cases; the others can be analyzed similarly. Note also that if one or two of the pseudoangles are trivial, then instead of the geodesic hexagon we obtain a pentagon or a quadrangle, which leaves us with fewer cases to consider.

For an arbitrary path $q$ in the Cayley graph of $H$, we denote by $\widetilde{q}$ the geodesic path with the same initial and terminal points as $q$. If $P_{1}$ is the projection of a point $P$ onto a path $q_{1}$ and $P_{2}$ is the projection of $P_{1}$ onto a path $q_{2}$, then we will call $P_{2}$ the projection of $P$ onto $q_{2}$ through $q_{1}$.

Case 1. (Fig. 10 a).) Let $A_{4}$ denote the projection of $A_{1} \in \widetilde{A B}$ onto $A B, C_{4}$ the projection of $C_{1} \in \widetilde{C D}$ onto $C D$ and $D_{4}$ the projection of $D_{1} \in \widetilde{E F}$ onto $E F$.

Claim: $A_{4} B$ is short. 
a)

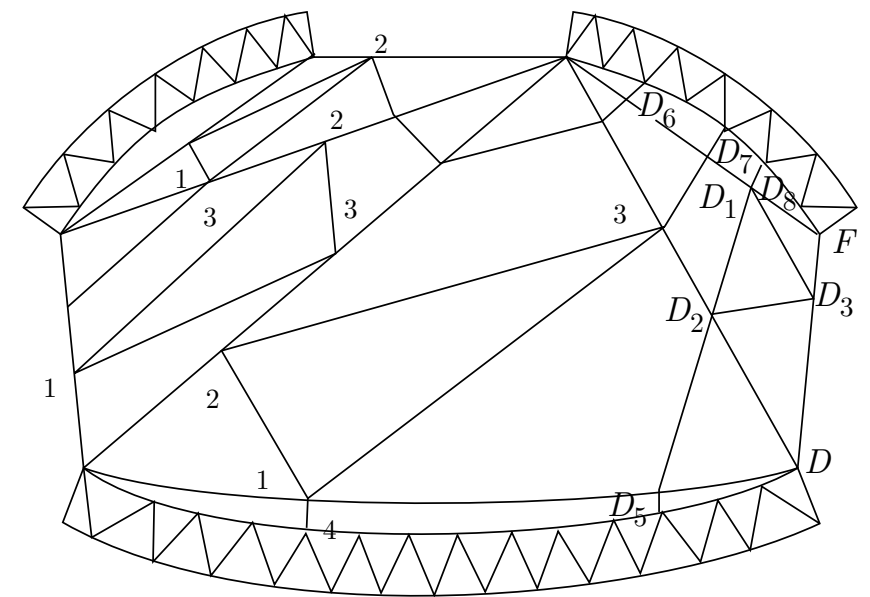

b)

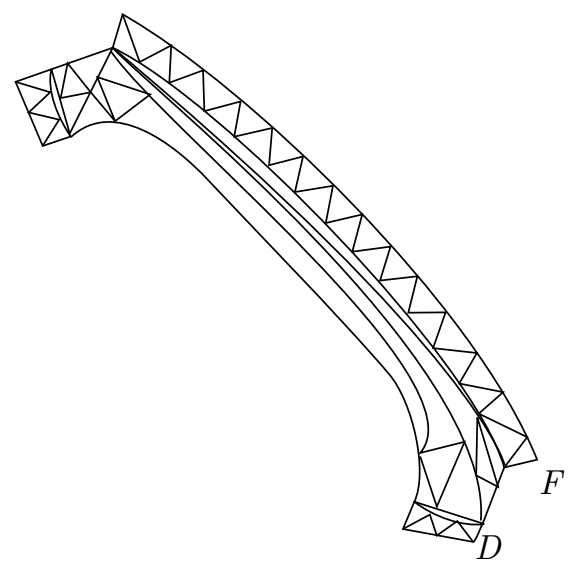

Figure 11

Proof. We may assume that $\left|A_{4} B\right|>M$ (the length of the connecting zone for $B E)$; otherwise there is nothing to prove. Let $p$ be the minimal path between $A B$ and $E F$ whose initial point is the farthest from $B ; \iota(p)$ is between $A_{4}$ and $B$. The path $B E$ is a connector, so its length is bounded by the following: $|B E| \leq|p|$ $+2 M$.

We now claim that $\left|B A_{2}\right| \leq\left|A_{4} A_{1} A_{2}\right|+2 M$. Indeed, suppose for contradiction that $\left|B A_{2}\right|>\left|A_{4} A_{1} A_{2}\right|+2 M$. Consider the path $B A_{2} E$.

$$
\begin{gathered}
|p|+2 M \geq\left|B A_{2} E\right|=\left|B A_{2}\right|+\left|A_{2} E\right| \\
>\left|A_{4} A_{1} A_{2}\right|+\left|A_{2} E\right|+2 M=\left|A_{4} A_{1} A_{2} E\right|
\end{gathered}
$$

It follows that $A_{4} A_{1} A_{2} E$ has length less than $|p|$, and therefore is a minimal path between $A B$ and $E F$, but its initial point falls outside the connecting zone for $B E$ - a contradiction. 
Therefore $\left|B A_{2}\right| \leq\left|A_{4} A_{1}\right|+\left|A_{1} A_{2}\right|+2 M \leq \mu+4 \delta+2 M$; by Lemma 1 $\left|A_{1} B\right|=\left|B A_{2}\right| \leq \mu+4 \delta+2 M$; finally, $\left|A_{4} B\right| \leq\left|A_{4} A_{1}\right|+\left|A_{1} A_{2}\right| \leq 2 \mu+4 \delta+2 M$, as required.

Claim: $A A_{4}$ is short.

Proof. Let $A_{5}$ denote the projection of $A_{3}$ onto $A B_{1}$; by the same argument as above $\left|A A_{5}\right| \leq A_{4} A_{1} A_{3} A_{5} \mid+2 M$ (otherwise $A C$ is not a connector). Consequently, we have: $\left|A A_{5}\right| \leq \mu+8 \delta+2 M$, and $\left|A A_{4}\right| \leq\left|A A_{5}\right|+\left|A_{4} A_{1} A_{3} A_{5}\right| \leq$ $2 \mu+16 \delta+2 M$.

Combining the two claims, we obtain that $A B$ is a short pseudoangle.

In the same way one can see that $C C_{5}, C_{4} D, F D_{4}$ and $D_{4} E$ are all short (here $C_{5}$ is $B_{3}$ projected onto $C D$ through them onto $C C_{1}$ ). However, it is not guaranteed that $C_{5} C_{4}$ is short, so it is possible for the pseudoangle $C D$ to be long. Fig. $10 \mathrm{~b}$ ) gives a more precise idea of what the middle looks like in this case.

As one can see from the calculations, $|A B|,|E F| \leq 4(\mu+10 \delta+M)$; denote this constant by $\nu_{1}$.

Case 2. (Fig. 11 a).)

Let $D_{5}$ denote the phase vertex closest to the projection of $D_{2}$ onto $C D$ through $D C_{1} ; C_{4}$ the phase vertex closest to the projection of $C_{1}$ onto $C D ; D_{6}$ the projection of $A_{2}$ onto $E F$ through $B_{2} E, B_{3} E, C_{3} E$ and $E D_{1}$.

In this case, $A B$ is short by the same argument as above; it is also clear that the following are short: $C C_{4}, D_{1} F, E D_{6}$ and $D D_{5}$.

Claim: $C_{4} D_{5}$ is short.

Proof. Let $D_{7} \in E F$ be the phase vertex closest to the projection of $C_{3}$ onto $E F$ through $E D_{1} ; D_{8}$ the phase vertex in $E F$ closest to the projection of $D_{1}$. Denote the path $C_{4} C_{1} C_{3} D_{7}$ by $p$ and $D_{5} D_{2} D_{1} D_{8}$ by $q$. Then by [1, Lemma 4] $\left|D_{7}, D_{8}\right|$, $\left|C_{4} D_{5}\right| \leq \max \left(M_{0},|p|,|q|\right)$ for some constant $M_{0}$. (Note that neither $v$ nor $w$ participates in $p$ or $q$.) Since $|p|$ and $|q|$ are bounded by $2 \max (|v|,|w|)+2 \mu+8 \delta$, this gives us a boundary on the length of $C_{4} D_{5}$.

$D_{6} D_{7}$ might turn out to be long. See Fig. $11 \mathrm{~b}$ ) for the shape of the middle in this case.

These calculations give us the constant $\nu_{2}$ bounding the lengths of the short pseudoangles $A B$ and $C D$ :

$$
\nu_{2}=32 \delta+4 \mu+8 M+\max \left(M_{0}, 2 \max \left\{\left|u_{1}\right|, \ldots,\left|u_{k_{1}}\right|\right\}+2 \mu+8 \delta\right) .
$$

In a similar way, one can compute the constants $\nu_{3}, \ldots, \nu_{8}$ bounding the lengths of the short pseudoangles in the remaining cases.

The following lemma can be proved by the same method as Lemma 5 .

Lemma 6. There are constants

$$
M_{1}=M_{1}\left(G, u_{1}, \ldots, u_{k_{1}}\right) \text { and } M_{2}=M_{2}\left(G, u_{1}, \ldots, u_{k_{1}}\right)
$$

such that for every middle $A B E F D C$ (Fig. 12) with a long pseudoangle $C D$ there exist points $P, Q \in B E$ and phase vertices $P_{1}, Q_{1} \in D C$ with the following properties:

1. $d\left(P, P_{1}\right)<M_{1}, d\left(Q, Q_{1}\right)<M_{1}$ and for all $P^{\prime} \in P Q$ we have $d\left(P^{\prime}, P_{1} Q_{1}\right)<$ $M_{1}$; 


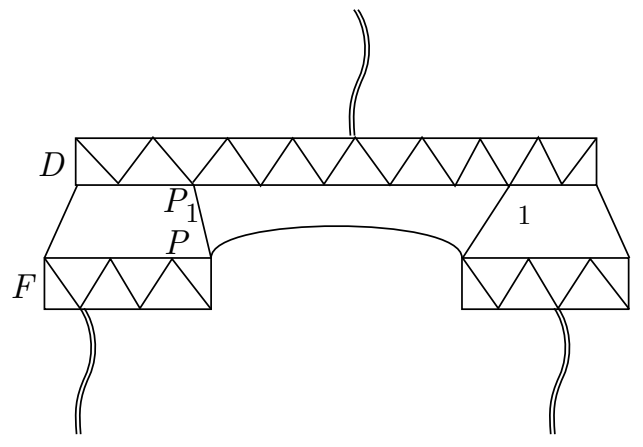

FIGURE 12

2. $\left|D P_{1}\right|,\left|Q_{1} C\right|<M_{2} \max \left(\left|u_{1}\right|, \ldots,\left|u_{k_{1}}\right|\right)$.

Proof. Let us take the same triangulation of the middle $A B E F D C$ as in the proof of Lemma 5. We will provide a proof for the case where the Gromov triangles inscribed in the triangles $A B E, A E C, C E D$ and $D E F$ are located with respect to each other as shown in Fig. 10 a); denote by $M_{1}^{1}$ and $M_{2}^{1}$ the constants satisfying the conditions of the lemma in this case. Similarly, one can consider the remaining seven cases to obtain the constants $M_{i}^{j}, i=1,2, j=2, \ldots, 8$. Then put $M_{1}=\max \left(M_{1}^{1}, \ldots, M_{1}^{8}\right)$, $M_{2}=\max \left(M_{2}^{1}, \ldots, M_{2}^{8}\right)$.

In the notation of Lemma 5 , let $P$ be the projection of $C_{2}$ onto $E A_{2}$ through $E B_{2}, Q$ the projection of $B_{2}$ onto $P A_{2}$, and $P_{1}, Q_{1}$ the phase vertices on $C D$ closest to $C_{4}$ and $C_{5}$, respectively. Then $d\left(P, P_{1}\right), d\left(Q, Q_{1}\right) \leq 12 \delta+\mu+\max \left(\left|u_{1}\right|, \ldots,\left|u_{k_{1}}\right|\right)$ $=M_{1}^{1}$.

In the same way as in the proof of Lemma 5 , one can see that $\left|C C_{5}\right|,\left|C_{4} D\right|$ $\leq 16 \delta+2 \mu+2 M$. Dividing this constant by $\max \left(\left|u_{1}\right|, \ldots,\left|u_{k_{1}}\right|\right)$, taking the integer part of the resulting number and adding 1 , we obtain the desired constant $M_{2}^{1}$.

\section{SHRINKING OF THE MIDDLE $t$-STRIPS}

Our next objective is to bound the powers of $u_{j}$ for $j=1, \ldots, k_{1}$ participating in the long pseudoangles. This will allow us to bound the number of triangular equations over the group $G$ obtained from the middles by triangulation. (For instance, the middle with the boundary label $\beta\left(s_{1}\right) \beta\left(u_{j}^{l}\right) \beta\left(s_{2}\right) \beta\left(s_{3}\right)$ shown in Fig. 13 produces $l+1$ triangular equations.)

Taking the middles of all equational triangles over the group $H$ corresponding to the system $S$, we obtain a system of equational diagrams; let us denote this

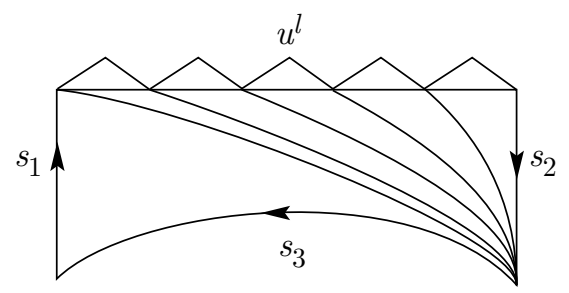

FIGURE 13 


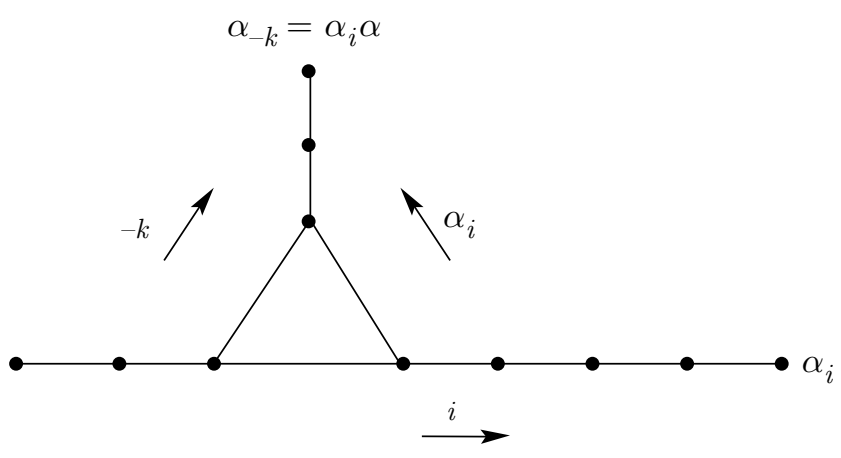

Figure 14

system by $\widetilde{S}$. Each diagram of the system $\widetilde{S}$ has the boundary label of the form $\beta\left(X_{1}\right) \ldots \beta\left(X_{l}\right)$. In what follows, it will be convenient to temporarily replace $\beta\left(X_{i}\right)$ for some of the $X_{i}$ by the canonical representative of $X_{i}$ in the sense of E. Rips and Z. Sela ([4]). (We will explain precisely for what $X_{i}$ we do this replacement after we define shrinkable words later in this section.) Let us recall the definition of Rips-Sela representatives, following the interpretation given by T. Delzant in [5].

Let $D$ be an integer, $\widetilde{S}:\left\langle a_{1}, \ldots, a_{n} ; R_{1}, \ldots, R_{T}\right\rangle$ a triangular system of equations in a torsion-free hyperbolic group $G$, and $\alpha_{1}, \ldots, \alpha_{n}$ a solution. Denote $a_{i}^{-1}=a_{-i}, \alpha_{i}^{-1}=\alpha_{-i}$; the equations are written in the form $\tau_{i, j, k}: a_{i} a_{j} a_{k}=1$, where $i, j, k \in\{ \pm 1, \ldots, \pm n\}$.

Definition 6. A family of $D$-canonical representatives in the sense of E. Rips and Z. Sela of $\alpha_{1}, \ldots, \alpha_{n}$ is a family of quasigeodesic polygonal paths $A_{i}(t)$ of length $L_{i}$ in the Caley graph $\Gamma(G)$ of $G\left(A_{i}(t):\left[0, L_{i}\right] \longrightarrow \Gamma(G), L_{i} \in \mathbf{N}, \alpha_{i}=A_{i}\left(L_{i}\right)\right)$ parametrized by arc length and satisfying the condition below.

Set $A_{-i}(t)=\alpha_{-i} A_{i}\left(L_{i}-t\right)$; let $\tau_{i j k}: a_{i} a_{j} a_{k}$ be one of the equations. Then the triangle $A_{i}, \alpha_{i} A_{j}, \alpha_{i} \alpha_{j} A_{k}$ is $D$-flat (see Figure 14) in the following sense:

$$
\begin{aligned}
& A_{i}(t)=A_{-k}(t) \quad \text { if } 0 \leq t \leq \frac{L_{i}+L_{k}-L_{j}}{2}-\frac{D}{2} \\
& A_{-i}(t)=A_{j}(t) \text { if } 0 \leq t \leq \frac{L_{i}+L_{j}-L_{k}}{2}-\frac{D}{2} \\
& A_{-j}(t)=A_{k}(t) \text { if } 0 \leq t \leq \frac{L_{j}+L_{k}-L_{i}}{2}-\frac{D}{2} .
\end{aligned}
$$

The paths $A_{i}$ are quasigeodesic, in other words,

$$
\frac{|t-u|}{v_{10 \delta}} \leq\left|A_{i}(t)-A_{i}(u)\right| \leq|t-u|(20 \delta+1),
$$

where $v_{r} \leq(2 N)^{r}$ denotes the number of elements in a ball of radius $r, N$ is the number of generators of $G$.

Let $\lambda^{\prime}$ denote the constant $1 / v_{10 \delta}$. It follows from Lemma 2 that there exists a constant $\mu^{\prime}=\mu^{\prime}\left(\delta, \lambda^{\prime}\right)$ such that for every geodesic word $\alpha$ and its Rips-Sela representative $A$ the inequalities $d(Q, A)<\mu^{\prime}$ and $d(P, \alpha)<\mu^{\prime}$ hold for any points $Q \in \alpha$ and $P \in A$. 
The main technical result of E. Rips and Z. Sela ([4]) consists in the following.

Theorem 1. [4] Let $T$ be a positive integer. Then there exists a number $D$ depending only on $T$ and the generators of $G$ such that for every system $\widetilde{S}$ of $T$ triangular equations over $G$, each solution $\alpha_{1}, \ldots, \alpha_{n}$ of $\widetilde{S}$ admits a family of $D$-canonical representatives.

Now let us return to the system $\widetilde{S}$ of equational diagrams over $G$ obtained by reduction from the original system $S$.

Definition 7. A middle $t_{j}$-strip is a $t_{j}$-strip whose $u_{j}$-side belongs to the long pseudoangle of a middle of an equational triangle.

Definition 8. A subword $w$ of the label of the boundary of a middle is said to be shrinkable if one of the following conditions is satisfied:

1. $w$ is the label of the $u$-side of a middle $t$-strip (e.g., $C D$ in Fig. 10 b);

2. $w$ is a connector such that a subword $v$ of $w$ is "close" to the $u$-side $q$ of a middle $t$-strip, meaning that for every point $P \in v d(P, q) \leq M_{1}+\mu^{\prime}$ (e.g., $B E$ in Fig. $10 \mathrm{~b}$ );

3. $w$ is a $t$-periodic word on the $t$-side of a middle $t$-strip;

4. if $w$ is the label of a common subpath of two paths $p$ and $p^{\prime}$ shrinkable as a subword of the label of $p$, then it is shrinkable as a subword of the label of $p^{\prime}$.

For each $j=1, \ldots, k_{1}$ and each middle $t_{j}$-strip, we will replace the power of $u_{j}$ on its boundary by a bounded power of $u_{j}$; during this procedure, the solution might be altered, but it will remain a solution of the original system of equations.

When we replace a $u_{j}$-word $w$ by another word, we should make appropriate changes to the diagram whose boundary contains the word $w$, as well as to the adjacent diagrams. Consider, for example, the diagram $A B C E D$ shown in Fig.15 a); here $C D$ is "close" to $A B$ in the sense of Definition 8. If we remove a subword $w$ from $A B$, we will also have to remove a subword of $C D$ and a subword of $D E$ (or $E C)$ together with some cells. Similarly, in the diagram $A B C F E D$ in Fig. 15b), removing a subword of $A B$ forces us to remove some subwords of $C D$ and $D E$ (or $C F$ ). In this diagram we assume that both $C D$ and $E F$ are close to $A B$, and $E F$ is a short pseudoangle of the diagram $C D E F$ (if it were long, it could not be close to $A B$ by Lemma 3 ).

For the sake of convenience, we will now denote by $\beta\left(X_{i}\right)$ the Rips-Sela representatives for the words $X_{i}$ that are on the boundary of triangular diagrams from the system $\widetilde{S}$ (e.g., $C E D$ from Fig. 15 a)) and the diagrams obtained by triangulation from polygonal diagrams containing no long pseudoangles ( $C D E F$ in Fig. 15 b)). The diagrams from Fig 15 a),b) now take shape shown in Fig 15 c),d) respectively.

Definition 9. Let $\sigma$ be the least integer greater than

$$
3 M_{2}+2 D / \max \left(\left|u_{1}\right|, \ldots,\left|u_{k_{1}}\right|\right) .
$$

The length $L(w)$ of a shrinkable word $w$ is defined as follows.

1. $L(w)=k-\sigma$ if $w=u_{j}^{k}, j \in\left\{1, \ldots, k_{1}\right\}$.

2. $L(w)=k-\sigma$ if $w=t_{j}^{s_{j} k+r}, 0<r<s_{j}, j \in\left\{1, \ldots, k_{1}\right\}$.

3. Let $w$ (or a subword of $w$ ) be close to the $u_{j}$-side $q$ of a middle $t_{j}$-strip in the sense of Definition 8. Denote by $P$ and $Q$ the phase vertices closest to the projections of $\iota(w)$ and $\tau(w)$ onto $q$, respectively; the path $P Q$ corresponds to a certain subword $u_{j}^{k}$ of $q$. Then we put $L(w)=k-\sigma$. 
a)

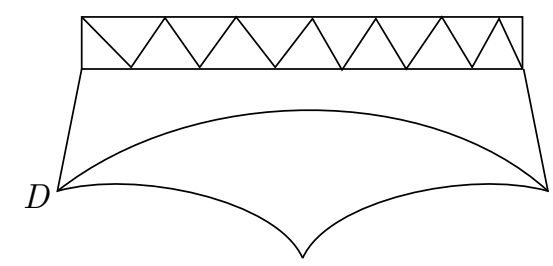

b)

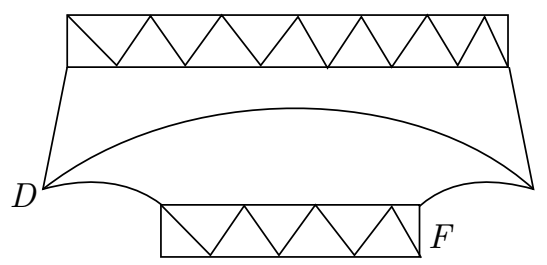

c)

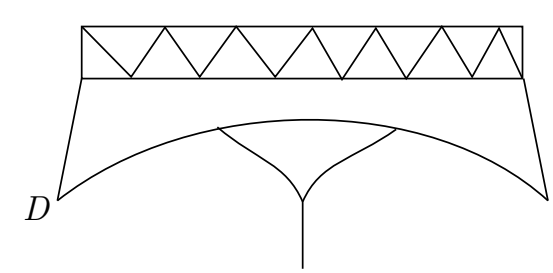

d)

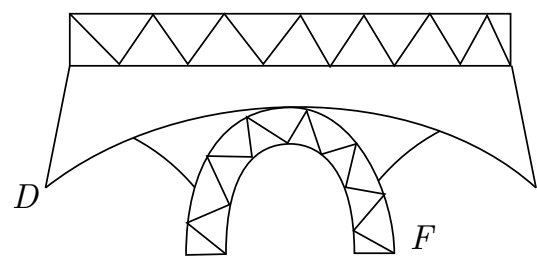

FiguRe 15

Definition 10. A maximal shrinkable subdiagram $\Delta$ of an equational triangle is the minimal subdiagram having three maximal shrinkable words on the boundary.

In Fig. 16 we illustrate all possible shapes of maximal shrinkable subdiagrams. (The diagram $A B C$ in Fig. $16 \mathrm{~d}$ ) is shrinkable since the path $A B$ is "close" to a $u$-side of a middle $t$-strip.)

Every maximal shrinkable subdiagram $\Delta_{k}$ gives us an equation over the integers as described below. Let $a_{k 1}, a_{k 2}$ and $a_{k 3}$ be the maximal shrinkable words on the boundary of $\Delta_{k}$ and let $u$ be the element from the set $\left\{u_{1}, \ldots, u_{k_{1}}\right\}$ used in the definition of length for the words $a_{k j}$; denote $L\left(a_{k j}\right)$ by $l_{k j}$. (We will be only interested in the case where at least one of $l_{k j}$ is positive, since we do not need 
a)

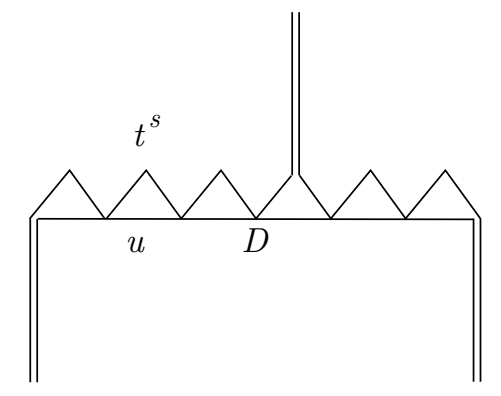

b)

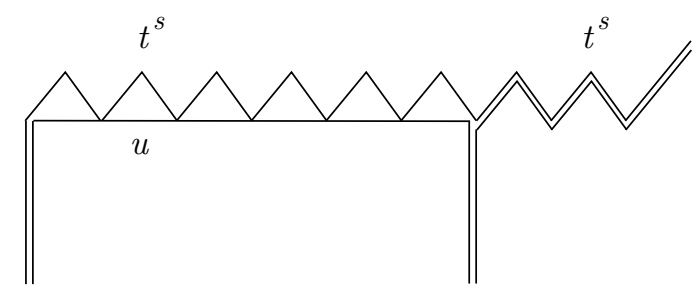

c)

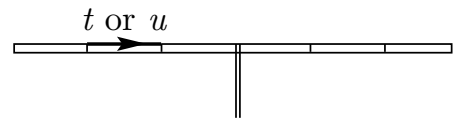

d)

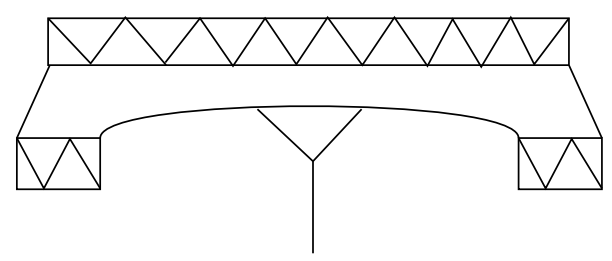

FiguRE 16

to "shrink" any words of nonpositive length.) Then to the subdiagram $\Delta_{k}$ we associate the equation $l_{k 1}+l_{k 2}+\sigma+s=l_{k_{3}}$, where $s \in\left\{0, \ldots, D^{\prime}\right\}$ and $D^{\prime}$ is the least integer greater than $D /|u|$. (For example, if $\Delta_{k}$ is the diagram shown in Fig. 16 a), then the $t_{j}$-word $A D$ is of length $l_{k 1}$, the $t_{j}$-word $E C$ of length $l_{k 2}$ and the $u_{j}$-word $A C$ of length $l_{k_{3}}$; we obtain the equation $l_{k 1}+l_{k 2}+\sigma+1=l_{k_{3}}$.)

We obtain an equation of this form from every maximal shrinkable subdiagram; denote the system of these equations by $L$.

It is possible to obtain only a finite number of distinct linear systems of this form. Every such system is algorithmically solvable, since the elementary theory of the natural numbers with addition is decidable. Choose arbitrarily a solution for each system. Let $l$ be the maximum of $l_{i j}$ in these solutions. Then the system $L$ also has a solution bounded by $l$. Let $\left\{\bar{l}_{i j} \mid 1 \leq i \leq m^{\prime}, 1 \leq j \leq 3\right\}$ be this solution. 
a)

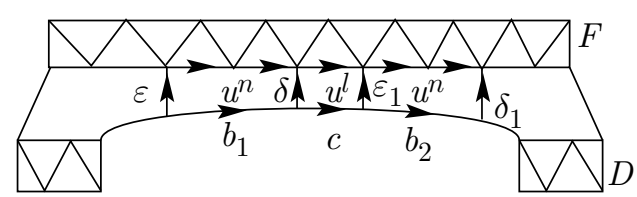

b)

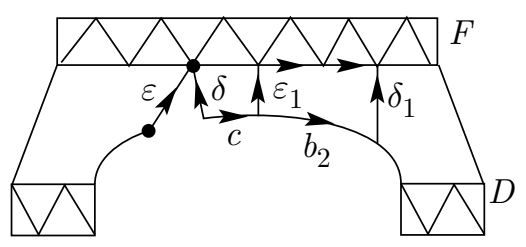

c)

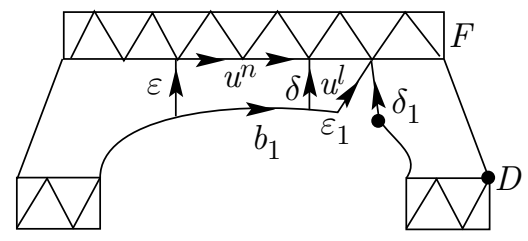

FigURE 17

We now replace each word $a_{k j}$, which has length $l_{k j}$, by a word $\bar{a}_{k j}$ of length $\bar{l}_{k j}$ as follows. If $a_{k j}=u^{l_{k j}+\sigma}$ for $u \in\left\{u_{1}, \ldots, u_{k_{1}}\right\}$, then $\bar{a}_{k j}=u^{\bar{l}_{k j}+\sigma}$; the same procedure applies if $a_{k j}$ is a power of $t \in\left\{t_{1}, \ldots, t_{k_{1}}\right\}$. Now let $a_{k j}$ be a maximal shrinkable word of the third type, meaning that a subword of $a_{k j}$ is close to the $u$-side $q$ of a middle $t$-strip. Let $P$ be the initial point of the path corresponding to the word $a_{k j}$ and $Q$ its endpoint. Denote by $P^{\prime}$ and $Q^{\prime}$ the phase vertices closest to the projections of $P$ and $Q$ onto $q$, respectively; the path $P^{\prime} Q^{\prime}$ represents the subword $u^{l_{i j}+\sigma}$ of $q$. Replace an arbitrary entry of $u^{l_{i j}}$ in $P^{\prime} Q^{\prime}$ by $u^{\bar{l}_{i j}}$, making the appropriate changes to the diagram $P P^{\prime} Q^{\prime} Q$. Then $\bar{a}_{k j}$ is the word corresponding to the path $P Q$ after these changes.

Replacing all the words $a_{j k}$ by $\bar{a}_{j k}$, we again get a solution of the original system of equations (see [1, Lemma 11]).

When we cut a power of $u$ out of a long pseudoangle of a middle, we also have to cut the corresponding cells out of the middle. Here we should make sure that no matter where on the path $E F$ (Fig. 17) we cut out $u^{n}$, the effect on the word $B C$ will be the same. More precisely,

Lemma 7. In the diagram shown in Fig. 17 a), $\varepsilon \delta^{-1} c b_{2}=b_{1} c \varepsilon_{1} \delta_{1}^{-1}$ (in other words, after removing the first copy of $u^{n}$, we get the same result as after removing the second copy).

Proof. Indeed,

$$
\begin{gathered}
b_{1} c \varepsilon_{1}=\varepsilon u^{n} u^{l}=\varepsilon u^{n+l} \\
\delta^{-1} c b_{2}=u^{l+n} \delta_{1}^{-1}
\end{gathered}
$$


Multiplying (2) by $\varepsilon$ and replacing $\varepsilon u^{n+l}$ in the right-hand side by the right-hand side of (1), we obtain the required equality.

The proof of Main Theorem is now essentialy the same as that of [1, Theorem 1]. It has to be mentioned that the original system $S$ is finally reduced to a finite set $F$ of systems in the hyperbolic group $\widetilde{G}=G *\left\langle t_{1}, \ldots, t_{\psi(m)}\right\rangle$, with the restriction that some of the variables belong to subgroups generated by only a part of the generating system of $\widetilde{G}$ (i.e., $u_{k_{i}+1}, \ldots, u_{k_{i+1}} \in H_{i}$ ). This restriction, however, does not prevent us from applying the Rips-Sela method to test each system from the set $F$ for solvability. Indeed, if a system has a solution $X_{1}, \ldots, X_{L}$ with some $X_{j}$ belonging to the group $G *\left\langle t_{1}, \ldots, t_{i}\right\rangle$, then the Rips-Sela representative of $X_{j}$ is forced to be in the group $F *\left\langle t_{1}, \ldots, t_{i}\right\rangle$. (Here $F$ stands for the free group with the same generating system as $G$.) Therefore, in the Rips-Sela method, the system over the hyperbolic group with the restrictions described above gets reduced to a system over a free group with the same restrictions, which is algorithmically decidable by $[6]$.

\section{REFERENCES}

[1] O. Kharlampovich, A. Myasnikov. Equations in a free $\boldsymbol{Q}$-group. Trans. Amer. Math. Soc. 350 (1998), 947-974. CMP 98:05

[2] O. Kharlampovich, A. Myasnikov. Hyperbolic groups and free constructions. Trans. Amer. Math. Soc. 350 (1998), 571-613.

[3] A. Yu. Ol'shanskii. On residualing homomorphisms and G-subgroups of hyperbolic groups. International Journal of Algebra and Computation, 3(4):365-409, 1993. MR 94i:20069

[4] E. Rips and Z. Sela. Canonical representatives and equations in hyperbolic groups. Invent. Math., 120: 489-512, 1995. MR 96c:20053

[5] Thomas Delzant. Finitely presented subgroups of hyperbolic groups. (Preprint)

[6] G. S. Makanin. Equations in a free group. Math. USSR Izv., V. 21, 1983. MR 84m:20040

Department of Mathematics and Statistics, McGill University, 805 Sherbrooke St. West, Montreal, Quebec, H3A 2K6 Canada

E-mail address: olga@triples.math.mcgill.ca

Department of Mathematics and Statistics, McGill University, 805 Sherbrooke St. West, Montreal, Quebec, H3A 2K6, Canada

E-mail address: kate@triples.math.mcgill.ca

Department of Mathematics, City College (CUNy), Convent Ave. at 138th Street, New York, New York 10031-9100

E-mail address: alexei@jolly2.sci.ccny.cuny.edu 